\title{
RESEARCH PROGRESS ON THE FOUR-IN-ONE METHOD SYSTEM FOR SUSTAINABLE PROTECTION OF ARCHITECTURAL HERITAGE
}

\author{
Yu Chi ${ }^{1,3,4}$, Aiqun $\mathrm{Li}^{1,2,3}$, Miaole Hou ${ }^{1,3,4}$, Yang Deng ${ }^{2} *$ ChongChen Wang ${ }^{5}$ \\ ${ }^{1}$ Beijing Advanced Innovation Center for Future Urban Design, Beijing University of Civil Engineering and Architecture, Beijing \\ 100044, China; tycy3932@163.com (Y.C.); liaiqun@bucea.edu.cn (A.L.); houmiaole@ bucea.edu.cn (M.H.); \\ dengyang@bucea.edu.cn(Y.D.), wangchongchen@ bucea.edu.cn(C.W.) \\ ${ }^{2}$ School of Civil and Transportation Engineering, Beijing University of Civil Engineering and Architecture, Beijing 100044, China \\ ${ }^{3}$ School of Geomatics and Urban Spatial Informatics, Beijing University of Civil Engineering and Architecture, Beijing 100044, \\ China \\ ${ }^{4}$ Beijing Key Laboratory for Architectural Heritage Fine Reconstruction \& Health Monitoring, Beijing 100044, China \\ ${ }^{5}$ School of Environment and Energy Engineering, Beijing University of Civil Engineering and Architecture, Beijing 100044, China.
}

\section{Commission II, WG II/8}

KEY WORDS: Architectural heritage value and protection demand cognition, All-round information access technology, Architectural heritage monitoring and evaluation, Architectural heritage performance improvement and value continuation.

\begin{abstract}
:
With the development of social economy, the definition, scope and connotation of architectural heritage have also evolved. However, the architectural heritage is facing the threat of destruction and destruction. The sustainable protection of architectural heritage has become a major strategic need of the country to enhance cultural self-confidence. In the past century, the concept of architectural heritage protection has evolved many times. At present, preventive protection and research protection have become the consensus in the field of architectural heritage protection. The idea of preventive protection is to delay the destruction or extinction of architectural heritage by controlling the main factors that cause the changes in the ontology. Preventive protection is different from emergency protection after the destruction of architectural heritage. It mainly discovers and eliminates hidden dangers through scientific records, periodic inspection, systematic monitoring and routine maintenance. It emphasizes the analysis of damage evolution law through risk assessment and scientific monitoring and takes effective protection measures. Based on the concept of preventive protection and research protection, combining the accumulation of prior art development and protection practices ,this paper proposes a four-in-one sustainable preservation method system for architectural heritage. It mainly includes four aspects: architectural heritage value and protection demand cognition, all-round information access technology, architectural heritage monitoring and evaluation, architectural heritage performance improvement and value continuation.
\end{abstract}

\section{INTRODUCTION}

Since 1987, the six heritages of the Forbidden City, Zhoukoudian Site Museum, and the Great Wall have been listed on the World Heritage List for the first time. The "Applying for cultural heritage" boom has been launched throughout China. There are 52 World Heritage projects in China that located in the world second by 2017 (Jin, 2018). Because of the nonrenewable of architectural heritage, it is particularly important to protect it. Some scholars have proposed research on the sustainable protection and development of architectural heritage With the development of modern digital technology, scholars at home and abroad have carried out architectural heritage protection work based on various digital technologies for historical buildings in the field of architectural heritage protection. These technologies have their own advantages, corresponding limitations and problems. This paper will focus on the domestic and international research status of these technologies, and propose a four-in-one sustainable preservation method system for architectural heritage that based on the concept of preventive protection and research protection, combining the accumulation of prior art development and protection practices. It mainly includes four aspects: building heritage value and protection demand cognition, all-round information access technology, building heritage monitoring and evaluation, building heritage performance improvement and value continuation.

\section{RESEARCH BACKGROUND}

The architectural heritage was first proposed as a proper noun in European Charter of the Architectural Heritage that adopted by the Council of Ministers of the European Parliament in 1975. It mentions that not only the most important monumental buildings, but also the natural and artificial environment of minor buildings in the ancient towns and characteristic villages (Zhang, 2007). The Convention Concerning the Protection of the World Cultural and Natural Heritage proposed "cultural heritage" including cultural relics, architectural complexes and cultural sites as a category of cultural heritage that adopted at the 17th meeting of UNESCO in 1972 (Chen, 2011). With the development of cultural heritage, the connotation of architectural heritage has also evolved. In 2003, International Council on Monuments and Sites redefines architectural heritage as a building or group of buildings of historical value (ICOMOS Charter, 2003), as shown in Figure 1 and 2.

\footnotetext{
* Corresponding author
} 


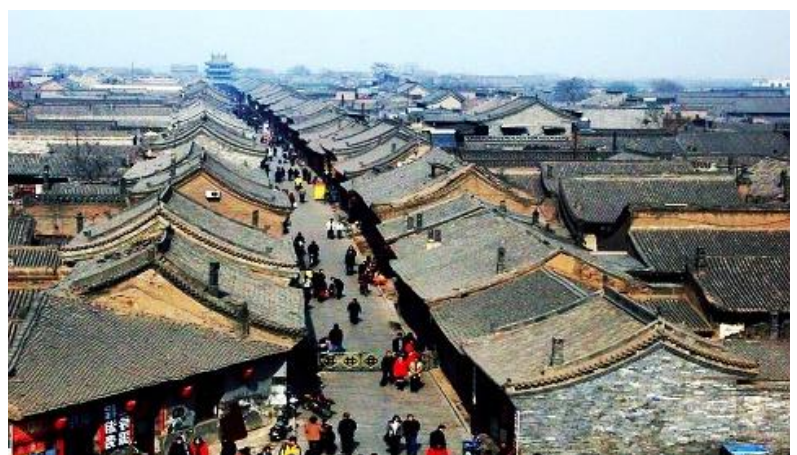

Figure 1. Historical town

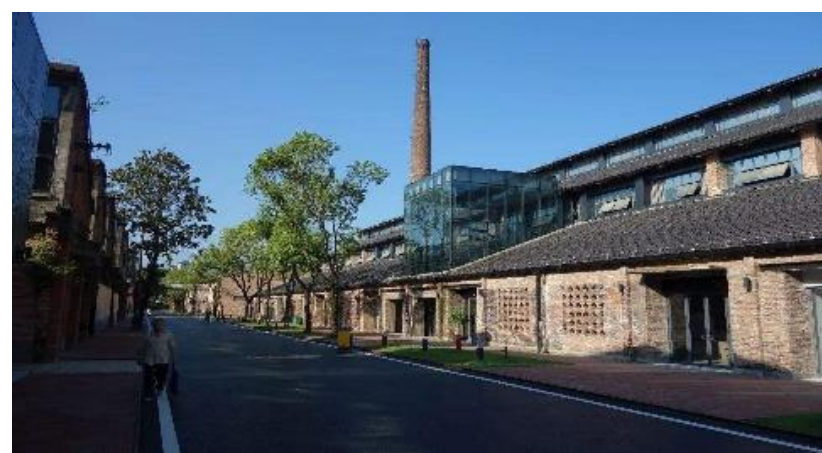

Figure 2. Industrial heritage

At present, the architectural heritage is facing the threat of destruction and extinction at home and abroad. For example, the half of wall of Colosseum has collapsed through the wind and rain, and some parts of the mantle are also exposed to the ground (Zhang, 2018).Although the overall preservation of Avalokitesvara is relatively complete. But it has been affected by wind ,sun and human activities, which makes its painted layer seriously eroded ( $\mathrm{Wu}$ and $\mathrm{Hu}, 2013)$, as shown in Figure 3.There are many factors that cause the destruction and demise of architectural heritage and need protection and repair. Therefore, it is particularly necessary to propose the sustainable development of architectural heritage that includes salvage protection, preventive protection and research protection. It has the comprehensiveness of architectural heritage protection and preserves the key features of the structure about history, art, culture and maintains ontology in the process of protection. In recent years, Chinese and foreign scholars have found that preventive protection is different from the emergency protection project after the destruction of building heritage. It regularly monitors and analyses the changes of the damage of the heritage structure and proposes various preventive measures that make the architectural heritage in a good state to avoid blindness. The protection project will ultimately achieve the sustainable development of building heritage protection ( $\mathrm{Wu}, 2011)$. The digital protection of architectural heritage can be better protected through the concept of sustainable protection in emergency protection, regular monitoring and expert discussion.

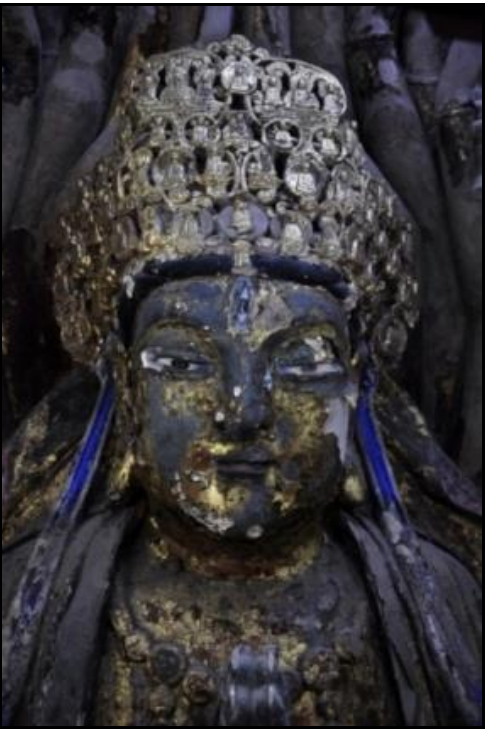

Figure 3. Avalokitesvara

\section{RESEARCH ROUTE}

Type text single-spaced, with one blank line between paragraphs and following headings. Start paragraphs flush with left margin.

\subsection{Cognition of Architectural Heritage Value and Protection Demand}

The types of architectural heritage values are diverse and complex, covering architectural, historical, archaeological, artistic, scientific and other values. Through the crossintegration and collaboration of knowledge in various disciplines, the value of architectural heritage can be accurately identified and evaluated. On this basis, accurately recognizing the protection needs of architectural heritage to formulate corresponding protection strategies is the premise of the four-inone method system for sustainable protection of architectural heritage.

Internationally, there are a sense of architectural heritage protection and a relevant protection charter. As early as 1933, the Congrès International d'Architecture Modern adopted the Athens Charter that the first international charter on the preservation and restoration of monuments. In 1964, the second meeting of the International Conference of Architects and Technicians adopted international Charter for the Conservation and Restoration of Monument and Sites in Venice. The Venice Charter is a principled document on the protection of cultural heritage under the Convention Concerning the Protection of the World Cultural and Natural Heritage, emphasizing the protection of the various values of protected objects, especially the protection of historical values. In 1981, the Washington Charter was the Charter for the Protection of Historic Towns and Cities, and supplemented the Venice Charter. These charters have had a great impact on China's protection of architectural heritage. China joined the World Heritage Convention in 1985 and participated in the World Heritage List in 1987. In the past 23 years, China has experienced tremendous changes from cultural relics protection to cultural heritage protection. The repeated repetition of the type of heritage reflects the deepening and expansion of the 
international community's understanding of cultural heritage that also reflected in the protection of Chinese cultural heritage. Through the continuous improvement of China's understanding of these new types of heritage in its own protection practices, China's cultural heritage protection system has also been continuously improved in this process(S, 2015).

\subsection{All-round information access technology}

With the rapid development of digital technologies such as manual mapping, photogrammetry technology and 3D laser scanning technology, digital information retention technology has been widely used in architectural heritage protection. The architectural heritage contains rich historical and cultural significance which cannot renewable and be immortal. It is significance to use digital technology to record and preserve its historical traces and existing state. With the rapid development of modern computer technology and laser technology, single traditional manual mapping and photogrammetry cannot accurately, stereoscopically and completely represent the threedimensional shape, structure and preservation of the heritage, so 3D laser scanning technology is more used in the heritage. The digital information retention of large complex architectural heritages fundamentally satisfies the non-contact, high-speed, high-precision, high-density, all-digital data collection requirements of the archaeological surveying and mapping field. Tan and Wang used remote sensing data to model the BeijingHangzhou Grand Canal, and combined the terrain model and the local landscape through aerial orthophotos to study the Beijing-Hangzhou Grand Canal (Tan and Wang, 2016). In the digital YunGang production and archaeological research, Lu and Ning used three-dimensional laser scanning and digital close-range photogrammetry technology to produce orthophoto images of the YunGang Grottoes (Lu and Ning, 2016). Hou and others used the articulated arm to obtain the point cloud data of the Avalokitesvara and made the model and then made a sectional drawing, an elevation drawing and a layout for later disease research [10]. Zheng and others studied the collaborative mapping mode in $2 \mathrm{D}$ and $3 \mathrm{D}$ environments, and used the contour automatic detection technology to sketch the elevation line of the facade and drew the elevation of the mural cave, which avoids the occlusion of the facade and the foreground(Zheng et al., 2016). Hou proposed the problem that the existing 3D scanning technology needs to solve in the study of the 3D model of the Avalokitesvara (Hou, 2017). Although digital access information technology has achieved a large number of high-fidelity results and applied to different types of architectural heritage. There are still a lot of information mining of massive data that has not deep enough and not yet provided a reasonable mechanical model for performance improvement and then need to be overcome.

On the basis of information retention, the HBIM model is built with digital information and historical legacy data which can provide engineering drawings for heritage restoration work and also provide a platform for health monitoring. Sheng and Yang found that based on the 3D laser point cloud data, Cloud Worx can connect to Cyclone software through AutoCAD to extract component point cloud data. Using CAD 3D modeling function to build the model, and merge and generate some components can overall geometric model of ancient buildings (Sheng and Yang, 2015). Because the data format of CAD and BIM is different from the acquired 3D point cloud, Qian et al. proposed to use STL format to convert 3D solid objects into 3D point clouds. After denoising and coarse registration, the KD tree is used to introduce ICP algorithm to have a fine registration.
Finally quantify the error by root mean square value RMS, and accurately detect the angle of error by Hough transform linear regression analysis method(Qian et al., 2016). Deng and others assisted the fusion of the top image of the building acquired by the aerial photography of the drone with based on the ground point cloud data acquired by 3D laser scanning technology that reconstructed the BIM model of the building in Revit to meet the need of small range for scenario construction(Deng et al., 2016).

Using the above technology to obtain the status information of the building heritage in digital form, it can provide reference and repair basis for virtual restoration, evaluation of monitoring and performance improvement. However, the degree of mining of massive digital information is still not deep enough to effectively serve the both performance evaluation and improvement of architectural heritage. All-round information retention technology research is the foundation of the four-inone methodology.

\subsection{Architectural heritage monitoring and evaluation}

As people's understanding and research on architectural heritage deepens, the idea of preventive protection is increasingly valued in the field of architectural heritage protection. The "rescue protection" and "preventive protection" were placed in the same important position by the "Twelfth Five-Year Plan" of the National Museum of China in the first time, and clearly pointing out the main task of the "Twelfth Five-Year Plan" is to "achieve organic combination of the rescue protection and preventive protection "(Bai et al., 2013).

When the concept of preventive protection has not been proposed, monitoring techniques have been used in the protection of building heritage. In 1979, Hangzhou Tiger Hill Tower established a scientific monitoring system to monitor tower tilt, tower settlement, ground subsidence and displacement around the tower (Chen, 2008). In 2007, sensors were installed in the basilica of the BaoGuo Temple to monitor the building environment, material changes, structural stress conditions and displacement deformation (Wu, 2012). Shi used distributed optical fiber monitoring to monitor the deformation and temperature of the wall of DongHua Gate of the Forbidden City, and arranged a fiber monitoring system to analyze the deformation and penetration of the wall that located two potential infiltration areas of the city platform (Shi, 2016). Jiang et al. used FBG strain sensor to study the deflection monitoring of wooden beams in ancient buildings and found that it is suitable for the strain increment caused by semi-rigid bearings (Jiang et al., 2016). Wang and others also used the FBG sensor and modified the sensor to be able to install on the surface of the wooden structure that support was customized and fixed with screws to monitor the wooden structure of the Tibetan ancient building (Wang,2010). Wang et al. used 3D laser scanning and photogrammetry to establish a 3D model, used Geomagic qualify for3D comparative analysis and used a total station to monitor the calculated azimuth combined with the $3 \mathrm{D}$ model for tilt analysis (Wang,2018). Toshikazu et al.. used the CCD imaging system to test the impact of natural disasters such as earthquakes and typhoons on the structure of the wooden tower of the Jojakkoji Temple in Kyoto (Toshikazu, 2010). It can be seen that the concept of monitoring and performance evaluation has already penetrated into the protection of building heritage. In the small shell project of Zhuhai Opera House, Wang used $\mathrm{C}$ to expand the IFC standard, and used the IFCbased monitoring information expression method to establish 
the model of sensor Revit, which realized the IFC expression and integration of monitoring information (Wang, 2015). Sun et al. designed a 3D visual monitoring system based on BIM to carry on real-time finite element analysis of building structures, which can accurately monitor major buildings in real time (Sun et al., 2017).

Monitoring thinking has formed a broad consensus in the field of architectural heritage protection. Through monitoring and testing techniques, the status of building heritage performance and environmental conditions can be perceived, and the evolution mechanism of disease or damage can be identified before the building is destroyed .Clearly influencing the law of environmental factors and obtaining scientific and reasonable performance evaluation results of architectural heritage. Although the monitoring concept has long been deeply rooted in people's minds, the problem of monitoring information fragmentation, discontinuity and low utilization still exists. At present, the government has not established a health monitoring index system for building heritage features and formed a systematic performance evaluation theory and method based on monitoring information. Building heritage monitoring and evaluation is the key to the four-in-one methodology.

\subsection{Architectural heritage performance improvement and value continuation}

The protection of architectural heritage is to extend its rich value for a long time. For a long time, in order to continue the cultural and artistic nature of architectural heritage, the comprehensive performance improvement of architectural heritage has begun to attract attention. It is comprehensive that it not only includes the restoration of diseases in murals, statues and paintings, but also the structural performance of ancient buildings, and focuses on improving its ability to resist disasters In addition to inheriting the traditional ancient building repair skills, it should also develop new structural reinforcement, reinforcement, isolation and shock absorption technology on the basis of both ensuring the safety and original features of the building to achieve the comprehensive performance of the building heritage.

Italy's earthquake-resistant legislation has increased the attention to historical buildings, and realized that the commonly using seismic strengthening measures will cause damage to the historical buildings and their structural unity. For the first time, the concept of structural "elevation" was introduced in the seismic strengthening of historical buildings to replace the previous "refurbishment" concept. According to the definition of the decree, "elevation" refers to the fact that while ensuring the safety of historical buildings, it does not essentially change the overall stress characteristics of the original structure (Lubitz, 1978).The restoration of Colosseum is based on the design concept of "repairing the old as the old" and not attempt to "restore the original appearance of the building". The bricks of different materials are used to construct the structure of the worn part, thus distinguishing the newly built part that protected the originality of the building, stabilized the building structure and completed the architectural form at the same time (Zhang, 2018). However, there is a controversial project in the performance improvement application, because copiny modern civil engineering reinforcement technology, the World Heritage Committee considered that the irreversible restoration project in 2010 has seriously affected the authenticity and integrity of the Bagrati Cathedral, it be included in the List of World Heritage in Danger. In order to eliminate the crisis of delisting of heritage, the post-repair project adopts new materials and new technologies to meet the needs of historical building reuse without violating the protection of cultural relics and monuments, and emphasizes the identifiability of interventions through the contrast between new and old (Du and Cao, 2018). It can be seen from the Bagrati Cathedral that the performance improvement technology needs to treat the existing architectural heritage more rigorously. The repair of the architectural heritage is not a completely new purpose, but is maintaining the original artistic and historical foundation on the basis of the existing Building materials to reinforce that in order to achieve the maximum continuation of the life and value of the building heritage. Cao used the sol-gel method to study the wooden components of the ancient building in the GuangKou Museum of the Tang Dynasty and the ancient building blocks of the Ming'an Dingkou Tower. From the investigation of the disease, using the sample wood with the corresponding antiseptic reagents and reinforce the reagents can protect the wood components (Cao, 2015). In summary, the improvement of architectural heritage performance and value continuation is the core issue of the four-in-one method system for practical application.

\section{CONCLUSION}

The use of the four-in-one system of sustainable preservation of architectural heritage can provide a more scientific and systematic protection. On the basis of understanding the value of architectural heritage, it is possible to accurately understand the protection needs of architectural heritage and formulate protection plans in a timely manner. At the same time, the digital technology can be used to obtain the status information of the heritage, and provide reference and repair basis for the next virtual restoration, monitoring and evaluation and performance improvement work. The four-in-one method system will be an important development direction for the sustainable development of architectural heritage.

\section{REFERENCES}

Bai, C.J., Han, X., Wu, C., 2013. Discussion on the Basic Problems of Building Heritage Deformation Monitoring under the Thought of Preventive Protection. Journal of Xi'an University of Architecture \& Technology (Social Science Edition), 2013, 32(02): pp. 54-58. DOI: 10.15986/j.10087192.2013.02.001.

Cao, J., 2015. Research on in-situ anti-corrosion and reinforcement of wood components in ancient buildings. Shaanxi Normal University.

Chen, R., 2008. Suzhou Yunyan Temple Tower Maintenance and Reinforcement Project Report. Cultural Relics Press, 2008.

Chen, X., 2011. The Trend of Contemporary International Architectural Heritage Protection Theory. Architect, 2011 (01), pp. 96-101.

Deng, L.J., Cheng, X.J., Cheng, X.L., et al., 2016. A BIM Model Reconstruction Method Based on Point Cloud Data[J]. Geology and Mineral Resources Survey, 2016, 32(4), pp. 14-16. DOI: $10.16864 /$ j.cnki.dkch.2016.0062.

Du, Q., Cao, Y.K., 2018. World Heritage of Delisting Due to Rehabilitation - Review of the Rehabilitation of the Church of 
Bagration, Georgia. Heritage and Conservation Studies, 2018(1).

Hou, M.L., Jiang, L.L. Hu, Y.G., 2017. Research on 3D Model of Cultural Relics and Its Problems in Application. Research of Heritage and Protection, 2017, 2(01), pp. 82-88. DOI: 10.19490/j.cnki.issn2096-0913.2017.01.018.

Hou, M.L., Wu, Y.H., Zhang, X.Q., Hu, Y.G., Zhang, Y.M., 2014. Fine 3D Information Retention of Cultural Relics Based on Articulating Arm Scanning.Sciences of Conservation and Archaeology, 2014, 26(03), pp. 94-98. DOI: 10.16334/j.cnki.cn31-1652/k.2014.03.018.

ICOMOS Charter-Principles for the analysis, conservation and structural restoration of architectural heritage. Journal of Women s Health, 2003.

Jiang, S.F., Tang, W.J., Wu, M.H., et al., 2016. Deformation Monitoring Method of Ancient Building Wood Beam Based on FBG Strain Measurement. Earthquake Engineering and Engineering Vibration, 2016, 01(6), pp. 198-206. DOI: 10.13197/j.eeev.2016.06.198.jiangsf.023.

Jin, L., 2018. "China's 20th Century Architectural Heritage Project" inherits cultural self-confidence_-Taking Beijing's selected projects as an example. Architectural Design Management, 2018(2).

Logothetis, S., Delinasiou A., Styliandis E., 2015. Building Information Modeling For Cultural Heritage; a Review. 25th International CIPA Symposium 2015, 31 August 04 September 2015, Taipei.

Lubitz, R., 1987. The Italian economic crises of the 1970's. International Finance Discussion Papers, 1978.

Lu, J.W., Ning, B., 2016. Digital Exploration and Practice of Cultural Relics in Yungang Grottoes. Research of Heritage and Protection, 2016, 1(02), pp. 11-14. DOI: 10.19490/j.cnki.issn2096-0913.2016.02.003.

Qian, M., Ma, X.J., Bao, R.B.et al., 2016. Component defect detection technology based on 3D laser scanning and BIM. Journal of Computer Measurement and Control, 2016, 24(2), pp. 14-17. DOI: 10.16526/j.cnki.11-4762/tp.2016.02.004.

Sheng, D.X., Yang, Z.Q., 2015. Construction of BIM Geometric Model of Ancient Building Based on Laser Point Cloud Data. Surveying and Mapping Engineering, 2015(7), pp. 76-80. DOI: 10.19349/j.cnki.issn1006-7949.2015.07.019.

Shi, Y.L., 2016. A study on distributed optical fiber monitoring for platform of donghua gate in the forbidden city, Beijing. Nanjing University, 2016.

Sun, L., Xu, Z.Q., Jin, Q., 2017. Research on Integration Method of Structural Health Monitoring System Based on BIM Platform. Journal of Shenyang Jianzhu University: Social Science Edition, 2017(4), pp. 410-415.

Tan, J., Wang, S., 2006. Virtual Scene Construction of LargeScale Cultural Heritage: A Framework Initiated from the Case Study of the Grand Canal of China, 2016, 6(1).
Toshikazu, H., Chikahiro, M., Yasushi, N., et al., 2010. Seismic and Wind Performance of Five-Storied Pagoda of Timber Heritage Structure. Advanced Materials Research, 2010, pp. 133-134:79-95.

Wang, C., 2015. Research of BIM-Based expression of monitoring information using IFC and integration method. Harbin Institute of Technology, 2015.

Wang, G.L., Wu, G.K., Wang, Y.M., et al., 2018. Multi-source data Gutta deformation monitoring research. Journal of Earth Information Science, 2018, 20(4), pp. 496-504.

Wang, J., Yang, N., Yang,Q.S., 2010. Structural Health Monitoring System for Heritage Buildings. Journal of Beijing Jiaotong University, 2010, 34(1), pp. 100-104.

Wu, M.P., 2011. A New Concept of International Heritage Protection-Analysis of Preventive Protection of Architectural Heritage. Chinese Journal of Cultural Relics, 2011(02), pp. 90-95.

Wu, M.P., 2012. Discussion on the problem of building heritage monitoring under the concept of preventive protection. Ancient Architecture Technology, 2012(02), pp. 26-29. DOI: 10.13942/j.cnki.hzjz.2011.03.037.

Wu, Y.H., Hu, Y.G., 2013. Discussion on the Application of Data Acquisition and Virtual Restoration in Dazu Rock Carving Repair. Chinese Journal of Cultural Relics, 2013(03), pp. 33-36.

Zhang, X.J., 2018. A Discussion on the Practice of Italian Architectural Heritage Protection-Taking the Grand Corner of Rome as an Example. Architecture and Culture, 2018(1), pp. 195-196.

Zhang, S. 2007. International Charter for the Protection of Urban Cultural Heritage and Selection of Domestic Laws and Regulations. Tongji University Press, 2007.

Zheng, S.M., Xia, G.F., Hu, C.M., Zhang, L.H., 2016. Application of Ground Lidar Technology in Fine Mapping of Grottoes in Cave Temple. Laser Journal, 2016, 37(01), pp. 5-8. DOI: 10.14016/j.cnki.jgzz.2016.01.005. 